\title{
Silencing tumor necrosis factor-alpha in vitro from small interfering RNA-decorated titanium nanotube array can facilitate osteogenic differentiation of mesenchymal stem cells
}

\author{
This article was published in the following Dove Press journal: \\ International Journal of Nanomedicine \\ 14 July 2016 \\ Number of times this article has been viewed
}

\author{
Zhenlin Wangl,* \\ Zhiqiang $\mathrm{Hu}^{2, *}$ \\ Dawei Zhang ${ }^{3}$ \\ Mengchuan Zhuo' \\ Jiwei Cheng' \\ Xingping $\mathrm{Xu}^{\prime}$ \\ Yongming Xing' \\ Jie $\mathrm{Fan}^{2}$ \\ 'Department of Orthopaedics, \\ 2Department of Otorhinolaryngology, \\ No I I 3 Hospital of PLA, Ningbo, \\ ${ }^{3}$ Department of Orthopaedics, Xijing \\ Hospital of PLA, Xi'an, People's \\ Republic of China \\ *These authors contributed equally \\ to this work
}

\begin{abstract}
Titanium implants are known for their bone bonding ability. However, the osseointegration may be severely disturbed in the inflammation environment. In order to enhance osseointegration of the implant in an inflamed environment, the small interfering RNA (siRNA) targeting tumor necrosis factor alpha (TNF- $\alpha$ ) was used to functionalize titanium surface for gene silencing. The chitosan-tripolyphosphate-hyaluronate complexes were used to formulate nanoparticles (NPs) with siRNA, which were adsorbed directly by the anodized titanium surface. The surface characterization was analyzed by scanning electron microscope, atomic force microscopy, as well as contact angle measurement. The fluorescence microscope was used to monitor the degradation of the layer. The coculture system was established with mesenchymal stem cells (MSCs) grown directly on functionalized titanium surface and RAW264.7 cells (preactivated by lipopolysaccharide) grown upside in a transwell chamber. The transfection and knockdown efficiency of TNF- $\alpha$ in RAW264.7 cells were determined by fluorescence microscope, quantitative polymerase chain reaction, and enzyme-linked immunosorbent assay. The cytoskeleton and osteogenic differentiation of MSCs were also analyzed. Regular vertical aligned nanotubes ( $\sim 100 \mathrm{~nm}$ diameter and $\sim 300 \mathrm{~nm}$ length) were generated after anodization of polished titanium. After loading with NPs, the nanotubes were filled and covered by a layer of amorphous particles. The surface topography changed and wettability decreased after covering with NPs. As expected, a burst degradation of the film was observed, which could provide sufficient NPs in the released supernatant and result in transfection and knockdown effects in RAW264.7 cells. The cytoskeleton arrangement of MSCs was elongated and the osteogenic differentiation was also significantly improved on NPs loading surface. In conclusion, the siRNA decorated titanium implant could simultaneously suppress inflammation and improve osteogenesis, which may be suitable for peri-implant bone formation under inflammatory conditions.
\end{abstract}

Keywords: titanium, siRNA, TNF-alpha, nanoparticles, osteogenic differentiation

\section{Introduction}

Titanium (Ti) and its alloys are widely used as orthopedic implants due to their high mechanical strength and osseointegration ability. ${ }^{1}$ However, the osseointegration may be severely disturbed under inflammatory conditions such as osteoarthritis. ${ }^{2}$ The elevated inflammatory factors would not only influence the initial establishment of osseointegration but also the long-term maintenance. The most validated inflammatory cytokine is the tumor necrosis factor alpha (TNF- $\alpha)$, which is involved in the focal loss of cartilage in osteoarthritis. ${ }^{3}$ Aktas et al even suggested that the serum TNF- $\alpha$
Correspondence: Dawei Zhang Department of Orthopaedics, Xijing Hospital of PLA, No 127 Changle West Road, Xi'an 710032, People's Republic of China

Email daweiz56@I26.com 
levels may be used to indicate osteoarthritis progression. ${ }^{4}$ In order to improve osseointegration, a lot of research has been devoted to the Ti surface modification. ${ }^{5}$ Generally, there are three kinds of modification approaches, that is, physical (surface topography), chemical (chemical coating), and biological (biomolecules coating). Adhesion and differentiation of cells are closely related to the properties of the substrate they attach to. Not only do surface chemical elements and physical topography guide cell behaviors, crystallinity is also involved in cell adhesion modulation. ${ }^{6}$ However, the physical- and chemical-based surface modification mainly influence cell behaviors indirectly, whereas a coating of biomolecules can directly participate in the biological process, which may be more powerful in inducing bone formation. ${ }^{7,8}$ Here we hypothesized that TNF- $\alpha$ level can also be controlled with suitable surface coating and the bone formation around Ti implant may be enhanced spontaneously after TNF- $\alpha$ suppression.

RNA-interference (RNAi) is a crucial posttranscriptional gene silencing process mediated by synthetic small interfering RNAs (siRNAs) or microRNAs. ${ }^{9}$ Due to the high specificity and efficiency, siRNA may be an ideal candidate for target gene downregulation. ${ }^{10,11}$ Actually, in some studies, silencing TNF- $\alpha$ expression by siRNA has been tried as a potential therapy for inflammation. ${ }^{12,13}$ To downregulate TNF- $\alpha$ expression in arthritis, the siRNA targeting TNF- $\alpha$ has also been delivered systemically as nanoparticles (NPs). ${ }^{14}$ However, different siRNA delivery pathways may lead to different effects. Until now, delivery of siRNA targeting TNF- $\alpha$ from Ti surface has not been tried and the interrelationship between TNF- $\alpha$ and osteogenic differentiation has not been confirmed yet. Nevertheless, the modulation of Ti by miRNA or siRNA to facilitate osteogenic differentiation has been widely performed in previous publications. ${ }^{15,16} \mathrm{In}$ fact, delivery of siRNA from other scaffolds has also been reported in various publications. ${ }^{17,18}$ Consequently, modification of Ti implant with siRNA targeting TNF- $\alpha$ may also be reasonable.

In the meanwhile, the target of drugs silencing TNF- $\alpha$ and bone formation cells are different. Specifically, the TNF- $\alpha$ is secreted by activated monocytes while the bone formation relies on the osteogenic differentiation of bone marrow mesenchymal stem cells (MSCs) in the in vivo environment. In order to better simulate the in vivo environment, we established an indirect coculture system consisting of murine MSCs and murine monocyte line RAW264.7 cells to evaluate the influence of TNF- $\alpha$ on osteogenic differentiation. Briefly, the MSCs were grown directly on titania nanotubes (NTs) substrate modified by siRNA targeting

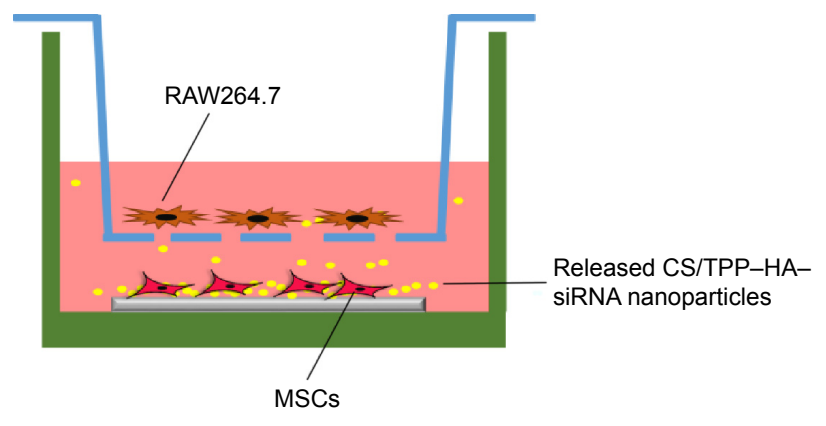

Figure I The coculture system illustration.

Abbreviations: CS/TPP-HA, chitosan-tripolyphosphate-hyaluronate; MSCs, mesenchymal stem cells; siRNAs, small interfering RNAs.

TNF- $\alpha$, which was placed at the bottom of the tissue culture plate. The RAW264.7 cells were grown upside in an inserted transwell chamber (Figure 1). The RAW264.7 cells were preactivated by lipopolysaccharide (LPS) to stimulate inflammatory environment. ${ }^{19}$ We hope that the inflammatory condition can be reduced by the released siRNA nanoparticles from the implant surface, thus promoting bone formation by reducing the negative impact of TNF- $\alpha$ on osteogenic differentiation of MSCs.

\section{Materials and methods}

\section{Titania NT fabrication}

Pure Ti foils $\left(99.8 \%\right.$ purity, $5 \times 5 \times 1 \mathrm{~mm}^{3}$, provided by Northwest Institute for Nonferrous Metal Research, People's Republic of China) were polished from 400 to 2,000 grid by $\mathrm{SiC}$ sandpaper and ultrasonically cleaned sequentially in acetone, absolute ethanol, and deionized water for 10 minutes each. The NTs were fabricated by anodization as described previously. ${ }^{20}$ Briefly, the cleaned Ti foil was anodized in $0.5 \mathrm{wt} \%$ hydrofluoric acid at $20 \mathrm{~V}$ for 30 minutes using a direct current power supply, followed by ultrasonic cleaning. The anodized sample was dried under nitrogen and sterilized by ultraviolet irradiation.

\section{NPs formulation}

The NPs were formulated by ionotropic gelation technique as previously described. ${ }^{21}$ Briefly, chitosan (CS; $150 \mathrm{kDa}$, 95\% deacetylation, MP Biomedicals, Santa Ana, CA, USA) was dissolved in hydrochloric acid $(0.04 \mathrm{M})$ overnight at a concentration of $2 \mathrm{mg} / \mathrm{mL}$. Tripolyphosphate (TPP) and sodium hyaluronate ( $\mathrm{HA}, \mathrm{Mw} \approx 360 \mathrm{kDa}$ ) were dissolved in deionized water ( $\mathrm{pH} 5.5$ ) at a concentration of $1 \mathrm{mg} / \mathrm{mL}$ and filtered through a $0.22 \mu \mathrm{m}$ pore size filter. The siRNA (target murine TNF- $\alpha$ sequence: sense, $5^{\prime}$-pGUCUCAGCCUCUU CUCAUUCCUGct-3', antisense, 5'-AGCAGGAAUGAG AAGAGGCUGAGACAU-3') was dissolved in RNase-free 
water at a concentration of $20 \mu \mathrm{M}$. To obtain the CS/TPP-HA NPs, the CS and TPP-HA solution were mixed at a volume ratio of 1:0.15:0.1 under magnetic stirring for 10 minutes. Then the $1 \mathrm{~mL}$ of CS/TPP-HA solution was mixed with $100 \mu \mathrm{L}$ siRNA and vortexed vigorously for 30 seconds to formulate the CS/TPP-HA-siRNA NPs. The size distribution was measured by dynamic light scattering-based Zetasizer (Malvern Instruments, Malvern, UK) to estimate the diameter. The morphology of particles was observed by transmission electron microscope (TEM, Tecnai, Hillsboro, OR, USA). In concisely, $4 \mu \mathrm{L}$ particle solution was dropped on a carbon-coated copper grid and air-dried to perform TEM observation. Three independent samples were examined and five fields of each sample were randomly selected to calculate the particle size by using ImageJ software (National Institutes of Health [NIH], Bethesda, MD, USA).

\section{Immobilization of NPs onto NT surface}

Due to the anodization procedure, the NTs surface exhibited a super-hydrophilic property. The formulated NPs aqueous was dropped directly onto NTs surface while shaking gently to spread the liquid homogeneously. The fabricated specimen was dried at $4^{\circ} \mathrm{C}$ overnight.

\section{Surface characterization}

The surface topography before and after coating with NPs was scanned by atomic force microscope (Veeco Instruments, Santa Barbara, CA, USA) under ambient conditions. The images were analyzed by using commercial Scanning Probe Image Processor (SPIP 6.0) software (Image Metrology ApS, Hørsholm, Denmark). Meanwhile, the water contact angle was analyzed with DSA100 drop shape analysis system (KRÜSS GmbH, Hamburg, Germany). The drop shape was photographed with a camera and the contact angle was calculated with the software provided by the manufacturer.

\section{Film degradation}

To determine the degradation of the film, CS was labeled by fluorescein isothiocyanate (FITC, MP Biomedicals) while the siRNA was labeled with cyanine 5 (Thermo Fisher Scientific, Waltham, MA, USA). Samples were exposed to phosphatebuffered saline (PBS) at $37^{\circ} \mathrm{C}$. After incubation for 0,1 , 3 , and 5 days, the sample was examined by fluorescence microscope (Olympus Corporation, Tokyo, Japan).

\section{Indirect coculture system}

The RAW264.7 cells and MSCs (C57BL/6; Gibico, Thermo Fisher Scientific, Waltham, MA, USA) were separately maintained in Dulbecco's Modified Eagle's Medium and $\alpha$-minimum essential medium supplemented with $10 \%$ fetal bovine serum and $1 \%$ penicillin/streptomycin. As illustrated in Figure 1, the activated RAW264.7 cells was seeded in the transwell chamber, while the MSCs were seeded on the implant, which was placed at the bottom of the well. Before the coculture, RAW264.7 cells was activated by LPS $(1 \mu \mathrm{g} / \mathrm{mL})$ for 4 hours according to a report elsewhere. ${ }^{22}$ Both cells were seeded at a density of 20,000 cells $/ \mathrm{cm}^{2}$. The study was judged exempt from ethic review by animal welfare committee of the Fourth Military Medical University, Xi'an, People's Republic of China under the relevant laws and institutional guidelines.

\section{Internalization of siRNA from released suspension}

The siRNA was labeled by Cy3 before fabrication of the NPs. After 24 hours of coculture, the transwell was collected and rinsed with PBS. Afterwards, the RAW264.7 cells was fixed by $4 \%$ paraformaldehyde (Beijing Leagene Biotech. Co, Ltd, Beijing, China) for 20 minutes. The nucleus was then stained with 4',6-diamidino-2-phenylindole and the cells were examined by inverted fluorescence microscope (Olympus Corporation).

\section{TNF- $\alpha$ determination}

The TNF- $\alpha$ expression of RAW264.7 cells was measured both at messenger RNA (mRNA) and protein levels. Briefly, after coculture for 48 hours, the total RNA of RAW264.7 cells was isolated by Trizol (Thermo Fisher Scientific, Waltham, MA, USA) and reverse transcribed. The determination of TNF- $\alpha$ mRNA was performed by real-time quantitative Taqman polymerase chain reaction. The TNF- $\alpha$ primer was: forward 5'-ATCCGCGACGTGGAACTG-3'; reverse $5^{\prime}$-ACCGCCTGGAGTTCTGGAA-3', and $\beta$-actin was: 5'-GCTCTGGCTCCTAGCACCAT-3' reverse 5'-GCCACCGATCCACACAGAGT-3'. The probe was FAM-CAGAAGAGGCACTCCCCCAAAAGATGGTAMRA. Meanwhile, the supernatant TNF- $\alpha$ concentration was measured by ELISA after 2 days of coculture. ${ }^{23}$

\section{Cytoskeleton staining}

The cytoskeleton of MSCs was examined after 2 days of coculture. Briefly, cells were fixed in 4\% paraformaldehyde for 15 minutes and treated with $0.1 \%$ Triton-X100 for 15 minutes. The actin filament was then stained with FITCconjugated phalloidin $(50 \mu \mathrm{g} / \mathrm{mL}$ in PBS) for 1 hour and rinsed with PBS three times. The nucleus was stained with 4',6-diamidino-2-phenylindole thereafter and specimens were observed by confocal laser scanning microscopy (Olympus Corporation). 


\section{Osteogenic differentiation}

The osteogenic differentiation of MSCs was determined by alkaline phosphatase (ALP) and collagen secretion. Briefly, MSCs on implant surface were induced in osteogenic medium, which was prepared in normal culture medium plus $10 \mathrm{mM} \beta$-glycerophosphate, $50 \mu \mathrm{g} / \mathrm{mL}$ ascorbic acid, and $100 \mathrm{nM}$ dexamethasone. The cells were fixed with $4 \%$ paraformaldehyde after incubation of the coculture system in osteogenic medium for 14 days. The ALP activity was determined by 5-Bromo-4-chloro-3-indolyl phosphate/ Nitro blue tetrazolium (BCIP/NBT) kit while the collagen production was stained by Sirius Red according to the manufacturer's instructions. After rinsing with abundant deionized water or $0.1 \mathrm{M}$ acetate acid to remove excess dye, the images were taken by stereomicroscope (Olympus Corporation). Afterwards, the color intensity was analyzed by Image J software.

Meanwhile, the total intracellular RNA was isolated by Trizol reagent (Invitrogen) and reverse transcribed by using the PrimeScript ${ }^{\mathrm{TM}} \mathrm{RT}$ reagent kit (Takara Bio, Inc., Shiga, Japan). The relative mRNA level of ALP (forward 5'-AACG

A

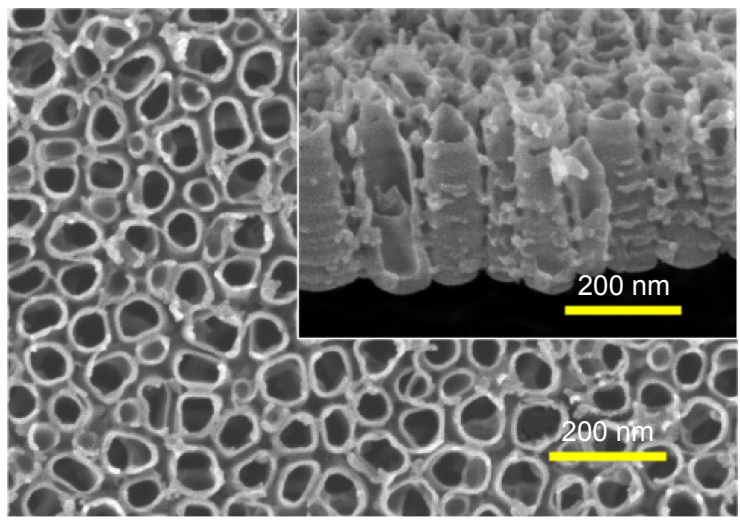

C

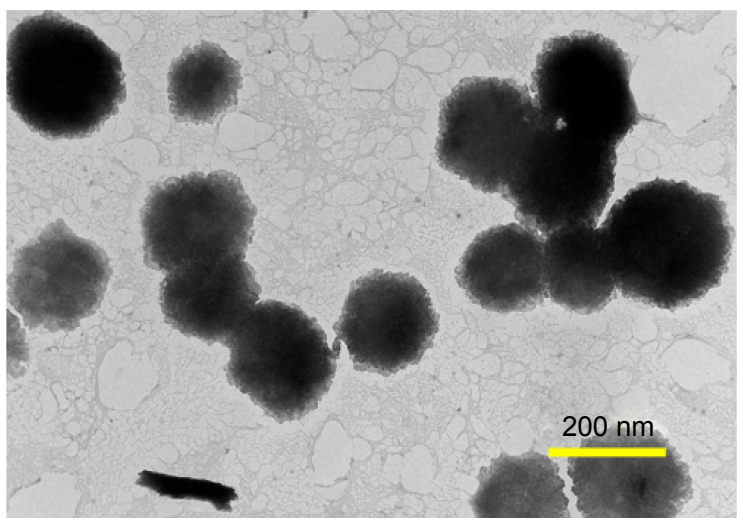

TGGCCAAGAACATCATCA-3', reverse 5'-TGTCC ATCTCCAGCCGTGTC-3'), and collagen type I $\alpha 1$ (COL1 $\alpha 1$, forward 5'-GCCTCCCAGAACATCACCTA-3', reverse 5'-GCAGGGACTTCTTGAGGTTG-3') were measured by SYBR Premix $\mathrm{Ex}^{\mathrm{TM}}$ Taq II on the Bio-Rad CFX96 qPCR System. GAPDH (forward 5'-GGCACAGTCAAGGCTGAGAATG-3', reverse ATGGTGGTGAAGACGCCAGTA-3') was used as an endogenous reference.

\section{Statistical analysis}

Three independent biological samples were repeated in each test and the results were presented as mean \pm standard deviation. One way analysis of variance and StudentNewman-Keuls-q test was used to compare the average and $P<0.05$ was considered significant.

\section{Results}

\section{CS/TPP-HA-siRNA NPs deposition}

Titania NTs with the diameter of $\sim 100 \mathrm{~nm}$ were fabricated (Figure 2A). From the cross-section view, the NTs length

B

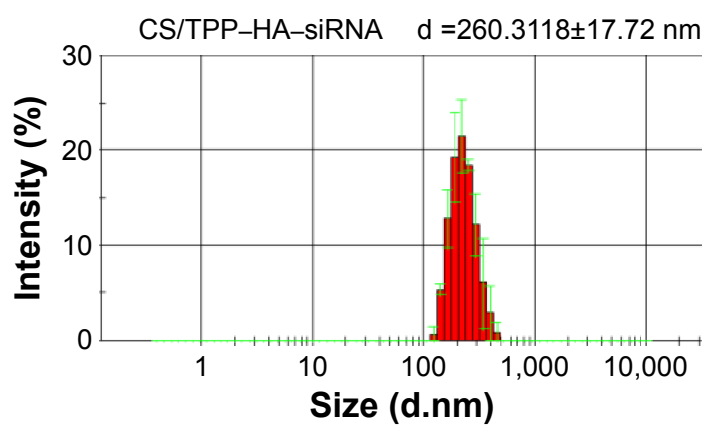

D

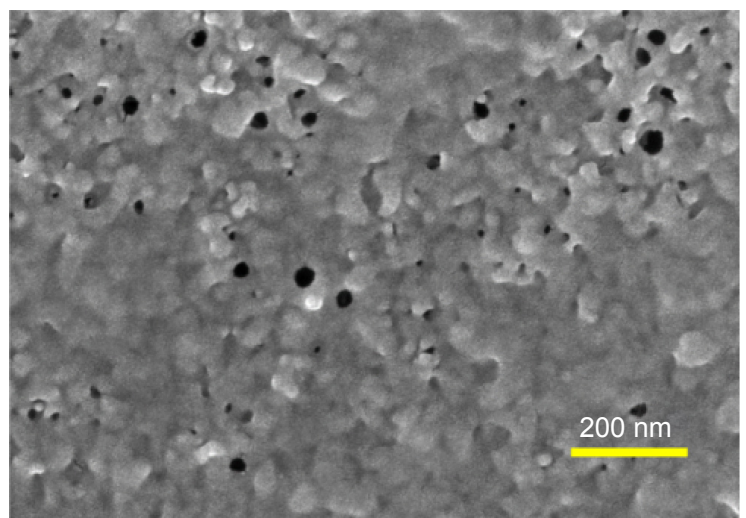

Figure 2 Implant and NPs observation by electron microscopy.

Notes: (A) The top and side view (inset) of titania NTs array by scanning electron microscopy observation (magnification 100,000). (B) Size distribution of CS/TPPHA-siRNA NPs measured by DLS. (C) The CS/TPP-HA-siRNA NPs morphology observation by transmission electron microscope (magnification I0,000). (D) The CS/TPPHA-siRNA NPs coating on NTs surface (magnification 100,000).

Abbreviations: CS/TPP-HA, chitosan-tripolyphosphate-hyaluronate; DLS, dynamic light scattering; NPs, nanoparticles; NTs, nanotubes; siRNAs, small interfering RNAs. 
was $\sim 250 \mathrm{~nm}$ and the surface of NTs array was ruffles-like (inset of Figure 2A). The CS/TPP-HA-siRNA NPs exhibited one peak at $\sim 260 \mathrm{~nm}$ (Figure $2 \mathrm{~B}$ ) the morphology observation by TEM showed a regular sphere shape (Figure 2C). After particles adsorption, it was clear that the NPs were aggregated around NTs surface to formulate an amorphous layer and it was hard to distinguish individual NPs (Figure 2D).

\section{Surface characterization}

The surface topography was scanned by atomic force microscopy. The NTs structures were still visible (Figure 3A1) but covered by irregular amorphous polymers (Figure 3A2). The water wettability was also measured (Figure 3B). The naked NTs were hydrophilic with the contact angle of $\sim 10^{\circ}$ while it increased to $\sim 45^{\circ}$ after coating with NPs (Figure $3 \mathrm{~B}$ ).

\section{Film degradation}

The degradation of the fabricated film was monitored by fluorescence microscope. With the increase of soaking duration, the fluorescence intensity of both CS and siRNA faded and their structures also collapsed (Figure 4). Most of the fluorescence faded during the first day of incubation (Figure 4), indicating a burst release of siRNA. In addition, the CS and siRNA were colocalized well on the implant surface (Figure 4).

\section{RAW264.7 cells transfection and inhibition}

The location of siRNA was tracked by fluorescence microscope after 24 hours of coculture (Figure 5A). The released siRNA had occupied the whole bottom of transwell, including intracellular space around the nucleus (Figure 5A). The TNF- $\alpha$ expression was significantly improved to nearly eight fold after activation with LPS (Figure 5B). However, approximately half of it was silenced by the released NPs (Figure 5B). Similar phenomenon could also be found in the mRNA of TNF- $\alpha$ (Figure $5 \mathrm{C}$ ).

\section{MSCs cytoskeleton}

The cytoskeleton of MSCs on Ti surface was revealed by FITC-conjugated phalloidin. On NPs loaded surface, the cells were elongated apparently, whereas they were spread as a polygonal shape on naked NT surface (Figure 6).

\section{Osteogenic differentiation}

The osteogenic differentiation of MSCs on implant surface was determined by ALP production and collagen secretion
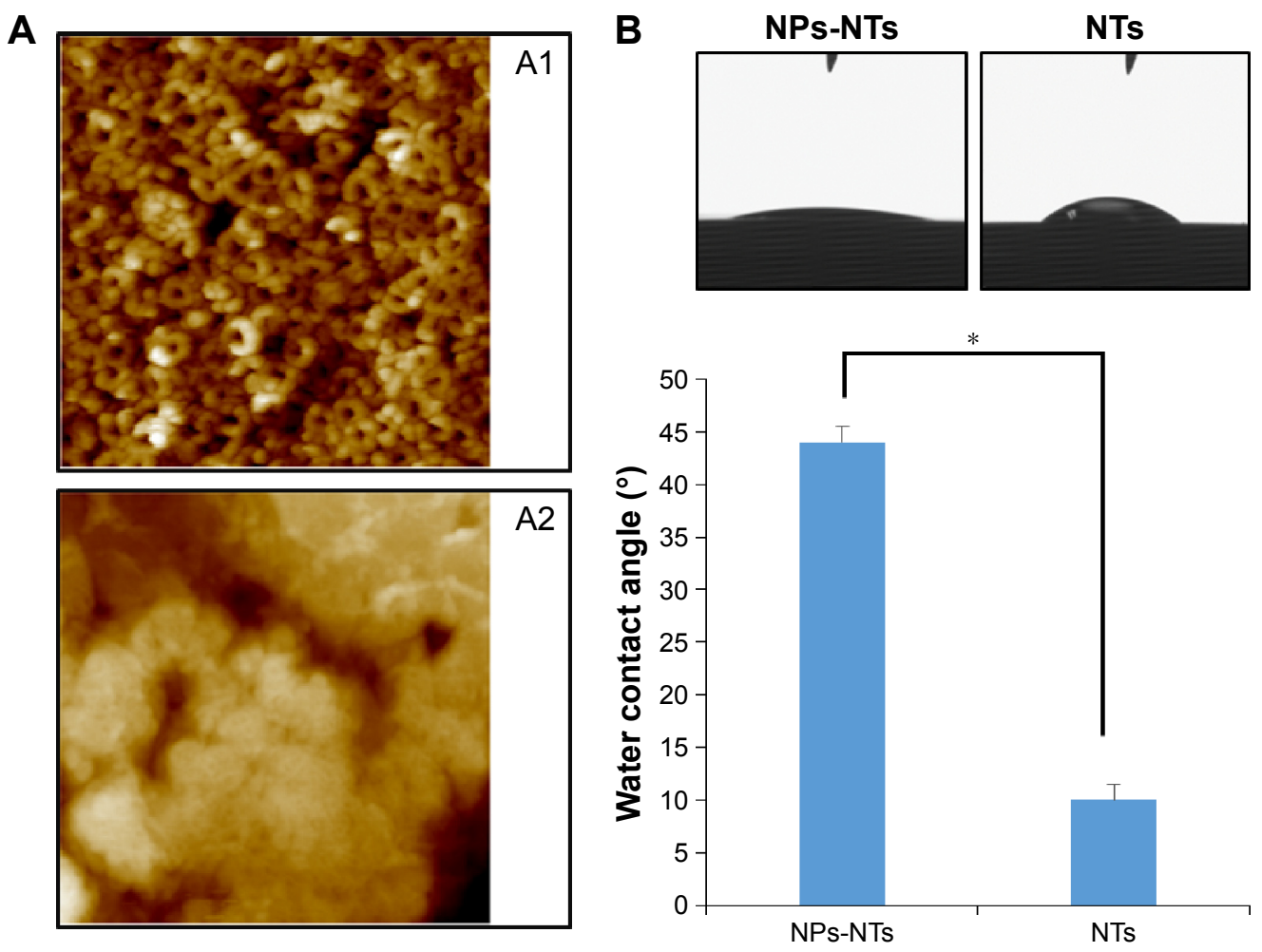

Figure 3 Surface topography scanning and water contact angle measurement.

Notes: (A) AFM images of naked NTs (AI) and NPs coating (A2). (B) Water contact angle measurement before and after NPs coating. $* P<0.05$.

Abbreviations: AFM, atomic force microscopy; NPs, nanoparticles; NTs, nanotubes. 

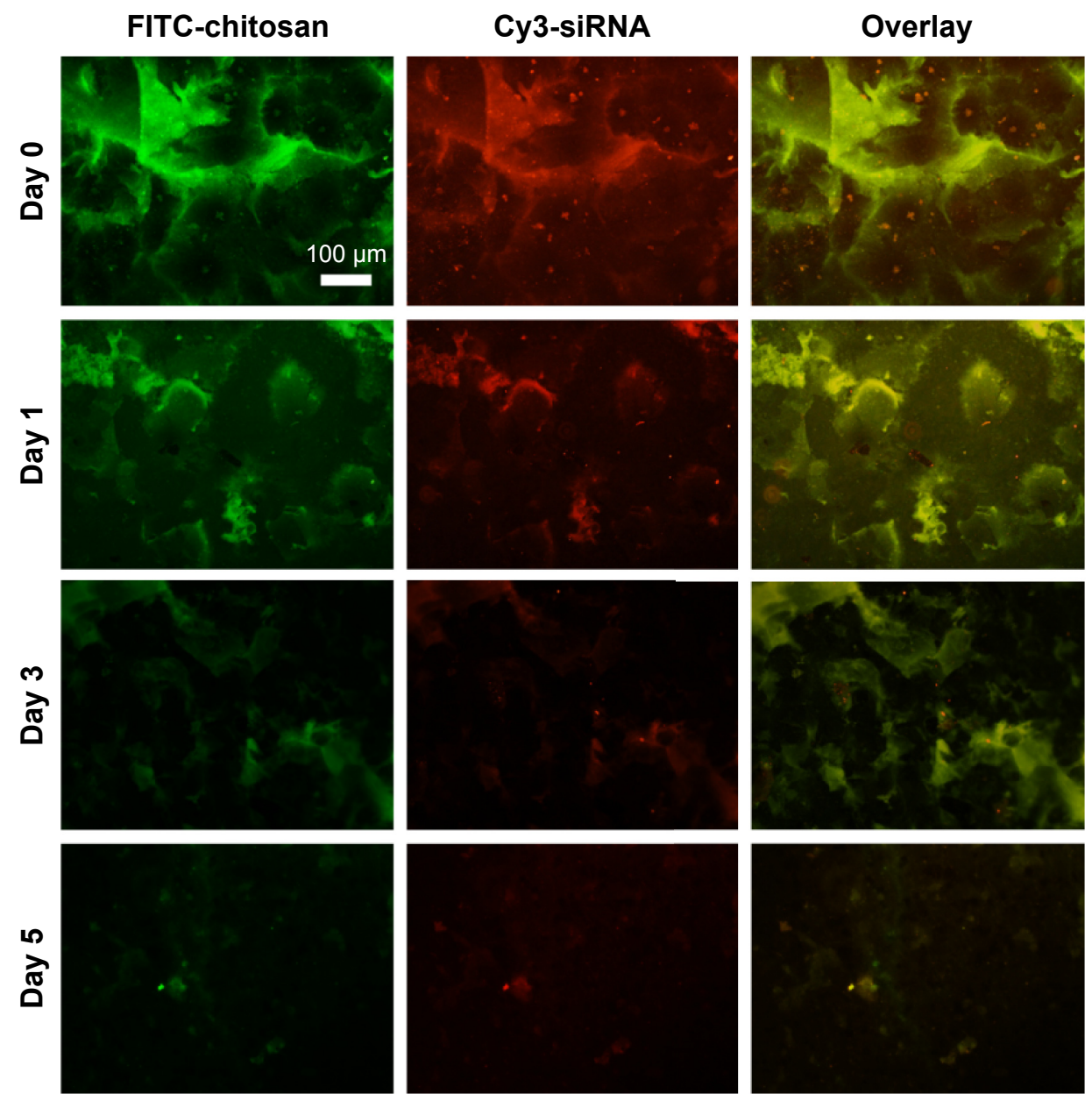

Figure 4 Degradation profile of the NPs coating in PBS

Notes: The chitosan was labeled by FITC while siRNA was labeled by $\mathrm{Cy} 3$ to formulate the NPs coating on NT surface. At the indicated incubation time, the implant surface was observed by fluorescence microscope. Scale $100 \mu \mathrm{m}(100 \times)$.

Abbreviations: FITC, fluorescein isothiocyanate; NPs, nanoparticles; NT, nanotube; PBS, phosphate-buffered saline; siRNA, small interfering RNA.

analysis. Both ALP activity and collagen secretion were significantly enhanced after loading with NPs (Figure 7). Specifically, a clustered ALP activity staining was obtained on NPs loaded surface (Figure 7A). Quantification of ALP activity showed approximately twofold increase on NPs loaded surface (Figure 7B). Similarly, the NPs -NT surface exhibited more intense collagen staining (Figure 7A), which was $\sim 1.5$ fold higher than the naked-NT surface (Figure $7 \mathrm{C}$ ). The relative mRNA level of ALP was more than sixfold higher and the osseous extracellular matrix specific collagen Type I was more than fivefold higher after loading with NPs (Figure 7D).

\section{Discussion}

Ti implants are widely used in the clinic. However, the peri-implant infection is still one of the most common complications that could seriously affect the osseointegration. Implant surface functionalization is extensively investigated to enhance osseointegration, among which the biomolecules coating may be more powerful due to the direct participation in biological process. The biofunctionalization with siRNA can directly target the specific molecular mechanism, which may be a powerful tool to improve the osseointegration in situ. In this study, CS/TPP-HA-siRNA NPs were developed. After anodization treatment of the Ti surface and physical adsorption of the CS/TPP-HA-siRNA complex, the functional implant could be fabricated with siRNA loading onto the Ti NTs surface. The target siRNA was introduced onto titania NTs surface by direct dip coating. The NTs structure formed by the anodization treatment could improve the surface area. Meanwhile, the titania NTs surface is superhydrophilic according to previous publications so that the aqueous NPs can be adsorbed easily on the surface. ${ }^{24,25}$

The CS/TPP-HA NPs have been recently proven to be a powerful vehicle for gene delivery, even to the spinal cord. ${ }^{21,26}$ In our study, the NPs also showed significant knockdown efficiency. The TPP-HA is negatively charged, which could facilitate the NPs formation with positively charged 

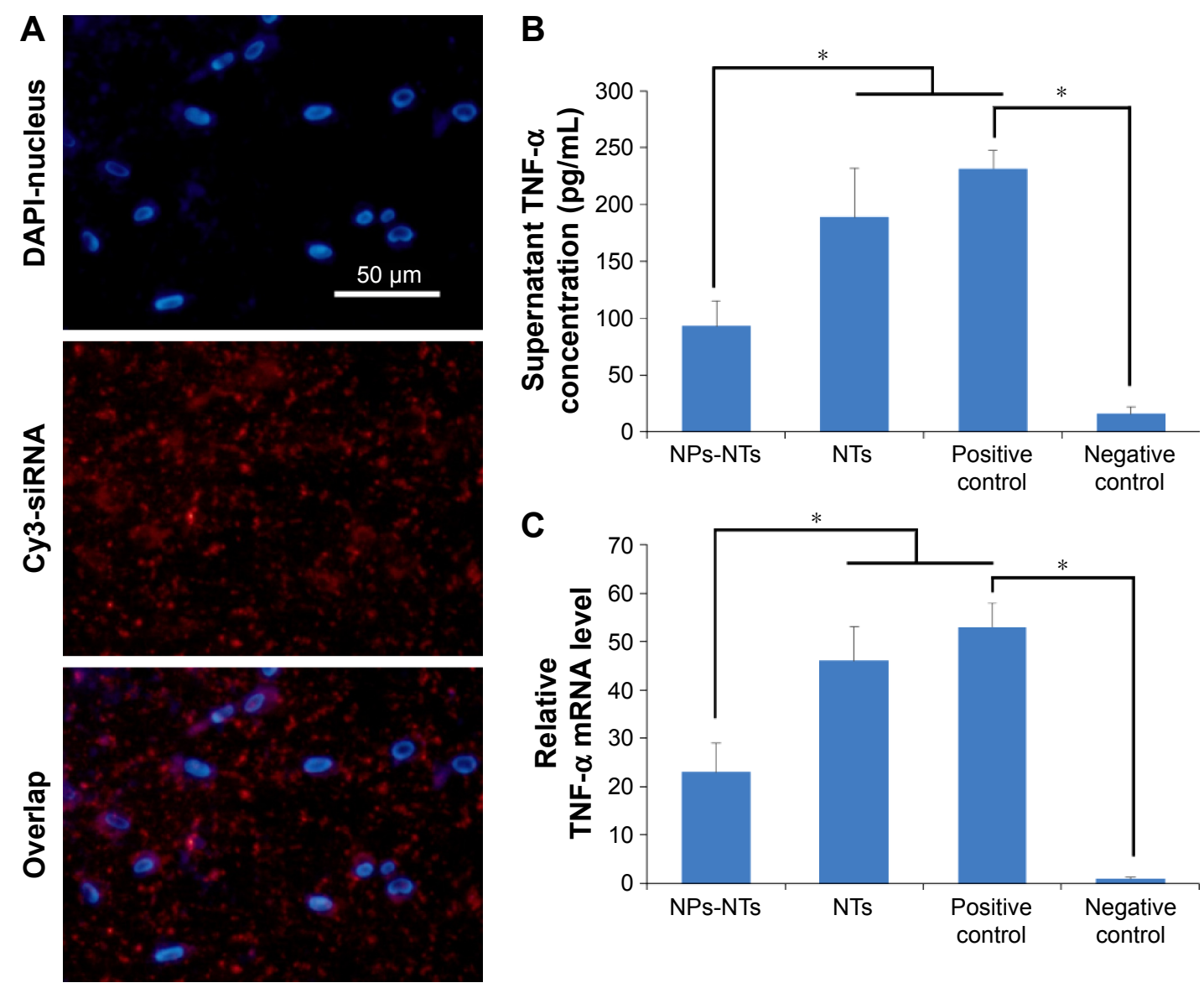

Figure 5 Transfection and knockdown efficiency of released NPs in RAW264.7 cells.

Notes: (A) Transfection efficiency observed by fluorescence microscope. (B) TNF- $\alpha$ secretion concentration in the supernatant quantified by enzyme-linked immunosorbent assay. (C) Intracellular TNF- $\alpha$ mRNA expression determined by Taqman polymerase chain reaction. $* P<0.05$. Scale $50 \mu \mathrm{m}(400 \times)$.

Abbreviations: DAPI, 4',6-diamidino-2-phenylindole; mRNA, messenger RNA; NPs, nanoparticles; NTs, nanotubes; siRNAs, small interfering RNAs; TNF- $\alpha$, tumor necrosis factor alpha.

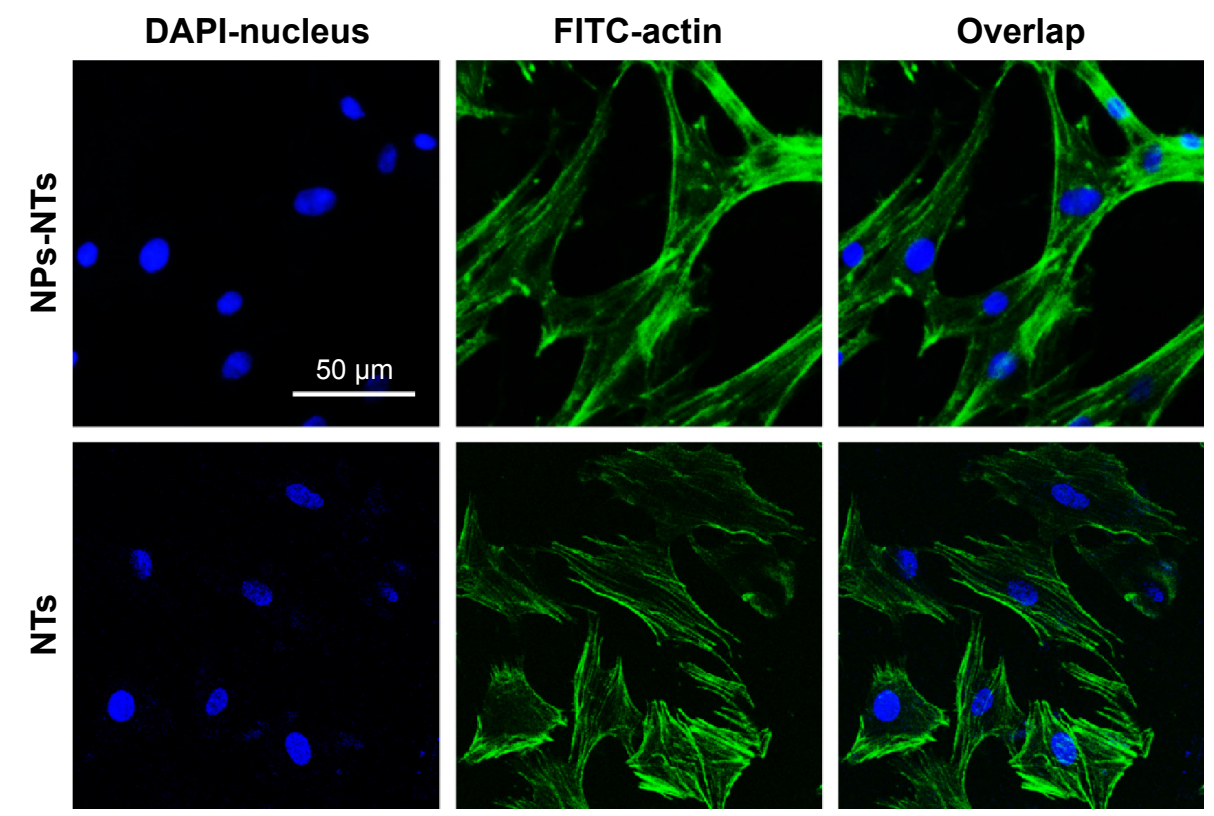

Figure 6 Cytoskeleton observation of mesenchymal stem cells growing on implant surface.

Notes: With or without NPs coating. Cells were fixed in $4 \%$ paraformaldehyde and dual stained with actin filaments and nucleus. Images were taken by confocal laser scanning microscope and the scope was randomly selected.

Abbreviations: DAPI, 4',6-diamidino-2-phenylindole; FITC, fluorescein isothiocyanate; NTs, nanotubes; NPs, nanoparticles. 

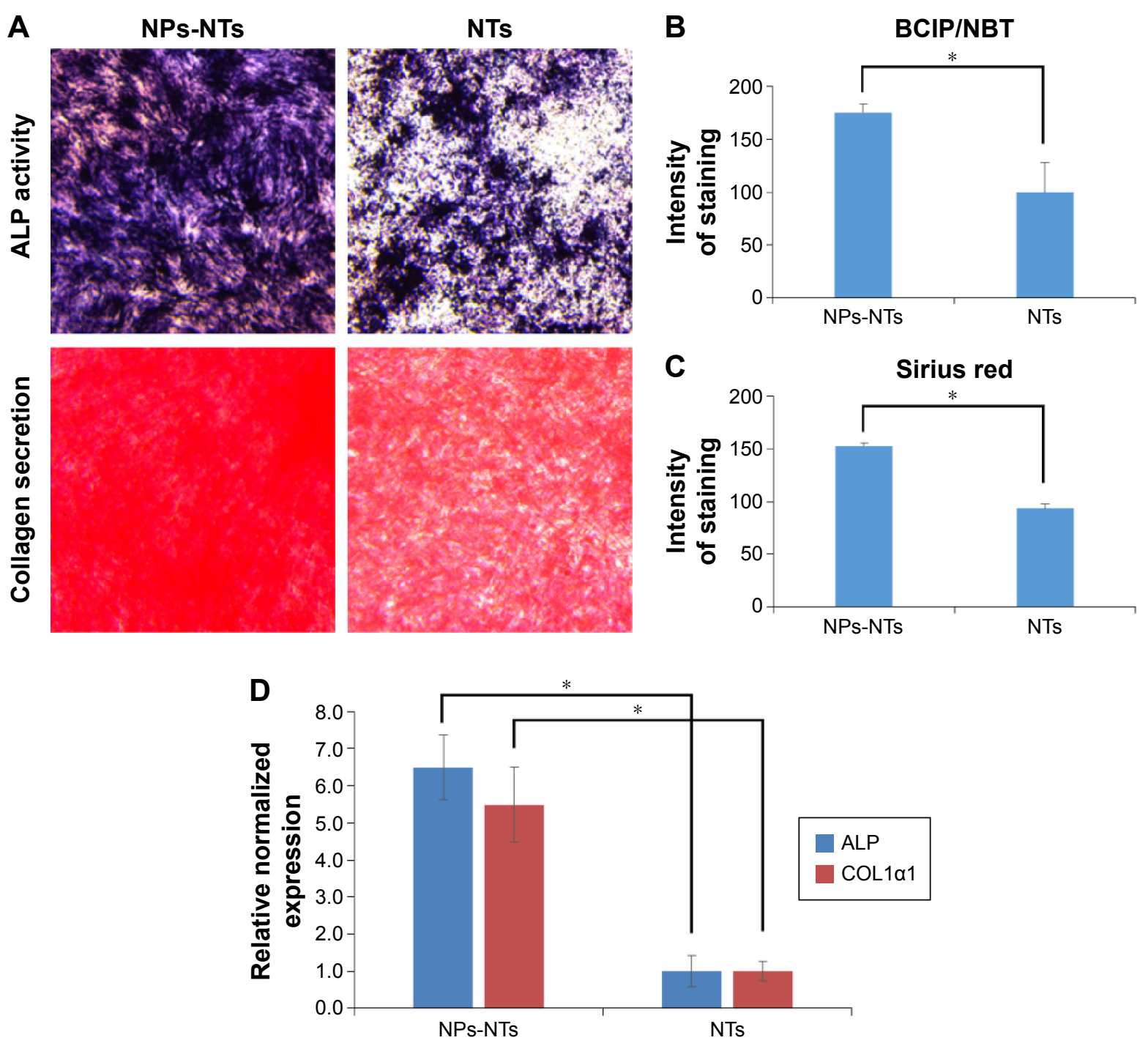

Figure 7 Osteogenic differentiation of mesenchymal stem cells on the titanium surface.

Notes: (A) ALP activity and collagen secretion staining. (B) Semiquantitative data of ALP activity. (C) Semiquantitative data of collagen secretion. (D) The relative messenger RNA level of ALP and COLI $\alpha$ I determined by quantitative polymerase chain reaction. $* P<0.05$.

Abbreviations: ALP, alkaline phosphatase; NTs, nanotubes; NPs, nanoparticles; COLI $\alpha$ I, collagen type I $\alpha$ I; BCIP/NBT, 5-Bromo-4-chloro-3-indolyl phosphate/Nitro blue tetrazolium.

CS/siRNA complex and help the NPs remain stable before application. However, the interaction between HA and CS are more complex according to Almalik A et al. ${ }^{27}$ From our observation, the NPs occupied most part of the NTs. The regularly aligned NTs surface was covered by an amorphous polymer and the hydrophilicity was significantly reduced due to the hydrophobic CS molecules. ${ }^{28}$ These indicate that the NPs are successfully deposited on the titania NTs surface. However, the simple adsorption cannot formulate a rigid interaction between the NPs and Ti. As a result, the burst degradation profile was observed. Interestingly, this may be crucial to guarantee the initial siRNA concentration for distant RAW264.7 cells transfection. Most RNAi functionalized scaffolds only focus on localized gene silencing in cells that are grown on them, so that the long term sustained siRNA release profile is the primary goal. ${ }^{29}$ However, in our case, the siRNA needs to work distantly in the inflammatory cells and in order to work distantly, the burst release system may be more suitable. RAW264.7 cells is macrophage-derived so that the uptake ability is fine. ${ }^{30}$ It was clear that the released siRNA had been internalized by RAW264.7 cells located upward on the transwell, indicating that the released siRNA was still able to be internalized by cells.

It is generally accepted that TNF- $\alpha$ is an inflammatory cytokine, which is an inhibitor of osteogenic differentiation of stem cells. ${ }^{31}$ However, there is also the opposite opinion that the TNF- $\alpha$ is able to promote osteogenic differentiation via activating nuclear factor kappa B signaling. ${ }^{32}$ These controversial conclusions may be attributed to the dosage of TNF- $\alpha$ treatment that lower concentration can promote 
while the higher concentration will inhibit osteogenic differentiation of stem cells. ${ }^{31}$ In our experiment, we use LPS to fabricate excessive inflammation environment, which was confirmed by dramatical upregulation of TNF- $\alpha$ expression. As expected, the osteogenic differentiation of MSCs on Ti surface was significantly attenuated during the coculture with activated RAW264.7 cells. However, after modification with siRNA targeting TNF- $\alpha$, the released NPs downregulated TNF- $\alpha$ expression considerably and the differentiation of MSCs was also improved. This indicates that the TNF- $\alpha$ concentration has been downregulated by siRNA to the level that is suitable for osteogenesis.

In conclusion, our present study demonstrates that the TNF- $\alpha$ secreted by activated RAW264.7 cells can significantly influence the osteogenic differentiation of MSCs. With siRNA decoration of Ti NTs surface, the TNF- $\alpha$ is downregulated distantly by the released NPs. After relieving of the inflammatory cytokine, the osteogenic differentiation of MSCs is improved spontaneously.

\section{Disclosure}

The author reports no conflicts of interest in this work.

\section{References}

1. Lugovskoy A, Lugovskoy S. Production of hydroxyapatite layers on the plasma electrolytically oxidized surface of titanium alloys. Mater Sci Eng C Mater Biol Appl. 2014;43:527-532.

2. Dekel N, Gnainsky Y, Granot I, Mor G. Inflammation and implantation. Am J Reprod Immunol. 2010;63(1):17-21.

3. Westacott CI, Barakat AF, Wood L, et al. Tumor necrosis factor alpha can contribute to focal loss of cartilage in osteoarthritis. Osteoarthritis Cartilage. 2000;8(3):213-221.

4. Aktas E, Sener E, Zengin O, Gocun P, Deveci M. Serum TNF-alpha levels: potential use to indicate osteoarthritis progression in a mechanically induced model. Eur J Orthop Surg Traumatol. 2012;22(2): $119-122$.

5. Liu X, Chu PK, Ding C. Surface modification of titanium, titanium alloys, and related materials for biomedical applications. Mat Sci Eng R. 2004;47(3-4):49-121.

6. Nelson M, Balasundaram G, Webster TJ. Increased osteoblast adhesion on nanoparticulate crystalline hydroxyapatite functionalized with KRSR. Int J Nanomedicine. 2006;1(3):339-349.

7. Lutz R, Srour S, Nonhoff J, Weisel T, Damien CJ, Schlegel KA. Biofunctionalization of titanium implants with a biomimetic active peptide ( $\mathrm{P}-15)$ promotes early osseointegration. Clin Oral Implants Res. 2010;21(7):726-734.

8. Chang YC, Feng SW, Huang HM, et al. Surface analysis of titanium biological modification with glow discharge. Clin Implant Dent Relat Res. 2015;17(3):469-475.

9. Agrawal N, Dasaradhi PVN, Mohmmed A, Malhotra P, Bhatnagar RK, Mukherjee SK. RNA interference: biology, mechanism, and applications. Microbiol Mol Biol Rev. 2003;67(4):657-685.

10. Dorsett Y, Tuschl T. siRNAs: applications in functional genomics and potential as therapeutics. Nat Rev Drug Discov. 2004;3(4): 318-329.

11. Elbashir SM, Harborth J, Lendeckel W, Yalcin A, Weber K, Tuschl T. Duplexes of 21-nucleotide RNAs mediate RNA interference in cultured mammalian cells. Nature. 2001;411(6836):494-498.
12. Kim SS, Ye C, Kumar P, et al. Targeted delivery of siRNA to macrophages for anti-inflammatory treatment. Mol Ther. 2010;18(5):993-1001.

13. Wilson DS, Dalmasso G, Wang L, Sitaraman SV, Merlin D, Murthy N. Orally delivered thioketal nanoparticles loaded with TNF- $\alpha$-siRNA target inflammation and inhibit gene expression in the intestines. Nat Mater. 2010;9(11):923-928.

14. Howard KA, Paludan SR, Behlke MA, Besenbacher F, Deleuran B, Kjems J. Chitosan/siRNA nanoparticle-mediated TNF-[alpha] knockdown in peritoneal macrophages for anti-inflammatory treatment in a murine arthritis model. Mol Ther. 2008;17(1):162-168.

15. Song W, Song X, Yang C, et al. Chitosan/siRNA functionalized titanium surface via a layer-by-layer approach for in vitro sustained gene silencing and osteogenic promotion. Int J Nanomedicine. 2015;10:2335-2346.

16. Wu K, Song W, Zhao L, et al. MicroRNA functionalized microporous titanium oxide surface by lyophilization with enhanced osteogenic activity. ACS Appl Mater Interfaces. 2013;5(7):2733-2744.

17. Liu X, Ma L, Gao C. RNAi functionalized scaffold for scarless skin regeneration. Organogenesis. 2013;9(2):76-78.

18. Andersen MO, Nygaard JV, Burns JS, et al. siRNA nanoparticle functionalization of nanostructured scaffolds enables controlled multilineage differentiation of stem cells. Mol Ther. 2010;18(11):2018-2027.

19. XuX, Yin P, Wan C, etal. Punicalagin inhibits inflammation in LPS-induced RAW264.7 macrophages via the suppression of TLR4-mediated MAPKs and NF-kappaB activation. Inflammation. 2014;37(3): 956-965.

20. Zhao L, Mei S, Wang W, Chu PK, Wu Z, Zhang Y. The role of sterilization in the cytocompatibility of titania nanotubes. Biomaterials. 2010; 31(8):2055-2063.

21. Gwak SJ, Jung JK, An SS, et al. Chitosan/TPP-hyaluronic acid nanoparticles: a new vehicle for gene delivery to the spinal cord. J Biomater Sci Polym Ed. 2012;23(11):1437-1450.

22. Tajima T, Murata T, Aritake K, et al. Lipopolysaccharide induces macrophage migration via prostaglandin D2 and prostaglandin E2. J Pharmacol Exp Ther. 2008;326(2):493-501.

23. Brembilla NC, Dufour AM, Alvarez M, et al. IL-22 capacitates dermal fibroblast responses to TNF in scleroderma. Ann Rheum Dis. Epub 2015 Oct 9.

24. Dong Hwan S, Tolou S, Chang Kyoung C, Seong-Hyuk L, Craig F. Wettability changes of $\mathrm{TiO}_{2}$ nanotube surfaces. Nanotechnology. 2011; 22(31):315704.

25. Ma Q-L, Zhao L-Z, Liu R-R, et al. Improved implant osseointegration of a nanostructured titanium surface via mediation of macrophage polarization. Biomaterials. 2014;35(37):9853-9867.

26. Nasti A, Zaki NM, de Leonardis P, et al. Chitosan/TPP and chitosan/ TPP-hyaluronic acid nanoparticles: systematic optimisation of the preparative process and preliminary biological evaluation. Pharm Res. 2009;26(8):1918-1930.

27. Almalik A, Donno R, Cadman CJ, Cellesi F, Day PJ, Tirelli N. Hyaluronic acid-coated chitosan nanoparticles: Molecular weightdependent effects on morphology and hyaluronic acid presentation. $J$ Control Release. 2013;172(3):1142-1150.

28. Ye J-R, Chen L, Zhang Y, Zhang Q-C, Shen Q. Turning the chitosan surface from hydrophilic to hydrophobic by layer-by-layer electroassembly. RSC Advances. 2014;4(102):58200-58203.

29. Rujitanaroj PO, Wang YC, Wang J, Chew SY. Nanofiber-mediated controlled release of siRNA complexes for long term gene-silencing applications. Biomaterials. 2011;32(25):5915-5923.

30. Wu C, Chen R, Liu M, et al. Spiromastixones Inhibit foam cell formation via regulation of cholesterol efflux and uptake in rAW264.7 macrophages. Mar Drugs. 2015;13(10):6352-6365.

31. Huang H, Zhao N, Xu X, et al. Dose-specific effects of tumor necrosis factor alpha on osteogenic differentiation of mesenchymal stem cells. Cell Prolif. 2011;44(5):420-427.

32. Hess K, Ushmorov A, Fiedler J, Brenner RE, Wirth T. TNFalpha promotes osteogenic differentiation of human mesenchymal stem cells by triggering the NF-kappaB signaling pathway. Bone. 2009;45(2): $367-376$. 


\section{Publish your work in this journal}

The International Journal of Nanomedicine is an international, peerreviewed journal focusing on the application of nanotechnology in diagnostics, therapeutics, and drug delivery systems throughout the biomedical field. This journal is indexed on PubMed Central, MedLine, CAS, SciSearch $\AA$, Current Contents $\AA /$ Clinical Medicine,

Journal Citation Reports/Science Edition, EMBase, Scopus and the Elsevier Bibliographic databases. The manuscript management system is completely online and includes a very quick and fair peer-review system, which is all easy to use. Visit http://www.dovepress.com/ testimonials.php to read real quotes from published authors.

Submit your manuscript here: http://www.dovepress.com/international-journal-of-nanomedicine-journal 